\title{
Perceptual averaging of line length: Effects of concurrent digit memory load
}

\author{
Ben Bauer ${ }^{1}$
}

Published online: 4 August 2017

(C) The Psychonomic Society, Inc. 2017

\begin{abstract}
The present experiment investigated performance in perceptual averaging of line ensembles during maintenance of minimal and near-span memory loads of digits. Observers memorized a four-to-seven digit number (high load) or a zero (low load) prior to a brief exposure $(500 \mathrm{~ms})$ of an ensemble of nine horizontal lines of various lengths. A subsequent probe line was then classified by observers as greater than or less than the ensemble average length followed by serial recall of the memory load. Slope analysis of the psychometric functions relating p("greater than") and the probe to ensemble-mean-size-ratio showed an advantage (steeper slope and therefore smaller threshold) for averaging under high-load compared with low-load conditions. Reaction time analysis indicated that faster probe responses were more accurate than slower responses.
\end{abstract}

Keywords Intuitive statistics - Averaging - Perceptual judgment $\cdot$ Dual task $\cdot$ Memory load

\section{Introduction}

Human observers demonstrate a striking ability to estimate the mean value of various properties from a group (ensemble) of visual stimuli (see, Alvarez, 2011; Bauer, 2015; Dakin, 2014; Haberman \& Whitney, 2012, for reviews). The extracted mean value is thought to be based on the contribution of many items, perhaps the whole ensemble or

Ben Bauer

benbauer@trentu.ca

1 Department of Psychology, Trent University Oshawa, Oshawa, ON L1J 5Y1, Canada a subset of items (Dakin, Bex, Cass, \& Watt, 2009; Simons \& Myczek, 2008; Solomon, Morgan, \& Chubb, 2011) and provides a compact summary of the property of interest.

One of the proposed processing benefits of summary statistics (see Alvarez, 2011) computed on visual images was well stated by Attneave (1954):

"It appears, then, that when some portion of the visual field contains a quantity of information grossly in excess of the observer's perceptual capacity, he treats those components of information which do not have redundant representation somewhat as a statistician treats 'error variance', averaging out particulars and abstracting certain statistical homogeneities." (p. 188).

Thus, data reduction may occur to prevent perceptual overload while usable summary information is retained to perform some task at hand (cf., Allik, Toom, Raidvee, Averin, \& Kreegipuu, 2013; Barlow, 2001).

In many studies, observers are given explicit instructions to perform perceptual averaging and to, for example, report which of two ensembles has a larger average, adjust a probe item to the average of the ensemble, or decide whether a probe is larger or smaller than the average. Recently, a spate of papers has provided good evidence that perceptual averaging occurs without explicit instructions to do so, and may in some sense be an obligatory and on-going property of the visual system (e.g., Allik, Toom, Raidvee, Averin, \& Kreegipuu, 2014; Dubé \& Sekuler, 2015), and the auditory system (e.g., Holt, 2006; Laing, Liu, Lotto, \& Holt 2012; Piazza, Sweeny, Wessel, Silver, \& Whitney, 2013), on multiple time scales (e.g., Dubé, Zhou, Kahana, \& Sekuler, 2014; Huang \& Sekuler, 2010) and over multiple frames of reference (e.g., Corbett \& Melcher, 2014). Further, perceptual averaging can take place inside and outside the focus of visual attention (e.g., Allik et al., 2014; Alvarez \& Oliva, 2008; Oriet $\&$ Brand, 2013). In fact based on their investigations of 
circle-size averaging, Allik et al. (2014) propose that "sizes of individual elements were pooled together in an obligatory manner before size information had reached awareness" (p.34).

The apparent ubiquity and robustness of the averaging process suggest that in some cases, it may not require central resources, at least at the computational level. Though the level(s) or stage(s) of processing at which visual averaging occurs remain undefined (see, Dubé \& Sekuler, 2015, footnote 1), a relatively early rather than late locus as suggested by Allik et al. (2014), Alvarez and Oliva (2008), and Dakin (2014), hints that averaging should survive a dual-task scenario provided that the nature of the additional task not contaminate the visual memory of the ensemble or its average. In fact, such contamination of visual memory by aveages of present and past stimuli has been one of the sources of evidence for the obligatoriness of perceptual averaging (e.g., Helson, 1947; Huang \& Sekuler, 2010). Thus, in the present experiment, perceptual averaging was the interpolated task between memorizing and serial recall of a four-to-seven digit number (high load) or a zero (low load). It is likely (though this was not tested) that the numeric task was verbally rather than visuo-spatially based (Aldred, Crawford, Duffy, \& Smith, 2016; Rissman, Gazzaley, \& D'Esposito, 2009) and therefore sufficiently different from the size averaging task to prevent contamination.

Several other pieces of evidence bear on this idea. First, in the early days of formal perceptual averaging research, (Anderson, 1964) found it useful to instruct observers "not to worry unduly about mathematical correctness but to give "what your feeling about the average is" (p. 191) and Spencer (1961) reported having to assure observers that perceptual averaging was even possible. Thus, using a dual-task procedure to tap into the perceptual process unencumbered by strategic and metacognitive influences might provide a more accurate profile of averaging processes. A recent version of this argument (Smilek, Enns, Eastwood, \& Merikle, 2006) supposed that coercing observers through instructions into a less hyper-vigilant mode of operation resulted in improved performance in a visual search task. Smilek, Enns, et al. (2006) encouraged observers to "relax" during visual search and this conveyed benefits in terms of slope reduction (lower time-cost per item) compared with when such encouragement was not provided (see also, Smilek, Dixon, \& Merikle, 2006; Watson, Brennan, Kingstone, \& Enns, 2010). Relatedly, Alvarez and Oliva (2008, 2009) demonstrated that background detection of ensemble changes is relatively unaffected by a foreground tracking task. In the present experiment, the goal is to positively affect ensemble processing rather than just show independence. Interestingly, Cocchi et al. (2011) report that a working memory load (positional) actually improved a perceptual grouping task in both speed and accuracy (see also, Bocanegra \& Hommel, 2014; Gil-Gómez de Liaño \& Botella, 2011).
The link here is that compelling the observers, through relaxing instructions or loading, to avoid micromanagement of the perceptual task may improve performance. One might notice an apparent paradox here given the body of research showing that attention improves performance on some perceptual tasks by essentially raising sensitivity (e.g., Anderson \& Druker, 2013; Gobell \& Carrasco, 2005; Pestilli \& Carrasco, 2005). However, Dubé, Zhou, Kahana, and Sekuler (2014) (see also, Dubé and Sekuler, 2015) suggest that in the case of perceptual averaging, the taxing of memory or reduction in observers' confidence in the precision of their ensemble memory (these are among the supposed byproducts of the dual task in the present experiment) "reliance on the perceptual averages is the greatest" (Dube \& Sekuler, 2015, p1).

It is possible that any gains shown in the present experiment are related to factors other than sensitivity per se. Once increased efficiency of perceptual averaging is demonstrated, the procedure can be modified to pinpoint the sources of this sharpening, such as encouraging a distributed rather than local attention mode (Baijal, Nakatani, van Leeuwen, \& Srinivasan, 2013; Chong \& Treisman, 2005a, b; Robitaille \& Harris, 2011), circumvention of extraneous influences in the observers' judgments such context and memory effects (e.g., Algom, Wolf, \& Bergman, 1985; Ross \& Di Lollo, 1971; Ward, 1987), or contraction bias (e.g., Ashourian, 2011; Hollingworth, 1910; Poulton, 1979).

Pulling these various considerations together, the goal of the present experiment was to determine whether averaging performance in the high-load condition would exceed performance in the low-load condition as indexed by a steeper psychometric slope and therefore smaller threshold under high-load compared with low-load conditions. Observers saw ensembles of nine horizontal lines of various lengths followed by a probe line that was $0.75,0.85,1.00,1.15$, or, 1.25 times the mean ensemble length. This occurred under conditions of high concurrent memory requirement (4-7 digits for serial recall) or low memory requirement (a single zero). Given that perceptual estimations are often considered to be based on 'snap judgments' (Fouriezos, Rubenfeld, \& Capstick, 2008), reaction time analysis will also be performed to determine the relationship between speed of responding and accuracy.

\section{Methods}

\section{Observers}

One hundred ninety-one observers from the undergraduate research pool participated for course credit. Each signed an informed consent form approved by the university research ethics board. The main analyses reported below are based on the 152 observers (unless otherwise noted) whose data 
passed the several inclusion criteria detailed in the Results section. For 76 observers, the high memory load condition included four or five digits for serial recall, and for the other 76, the high memory load condition included six or seven digits. Given that at the level of slope analysis each observer contributes a single value, which is a fitted parameter, a large number of observers were deemed necessary to overcome individual differences (see, Onyper, Zhang, $\&$ Howard, 2010). In addition, one of the reaction-time analyses below contrasts the speed of nominally correct vs. incorrect probe decisions based on the probe size to ensemble mean size ratio. A large sample size will help in providing sufficient numbers of rarer incorrect responses to make these comparisons more meaningful.

\section{Apparatus}

Presentation and response logging were under the control of an Apple PowerMac G4 (PPC 7455) driving an nVidia GeForce 4 MX (NV18) graphics card. The monitor was a LaCie Electron Blue IV 19" CRT running at $1152 \times 864$ pixel resolution and $119-\mathrm{Hz}$ vertical refresh rate. The experiment was programmed in Python using Pygame libraries running on PPC Linux 2.6.24.5. At an approximate viewing distance of $50 \mathrm{~cm}$, one pixel subtends $0.035^{\circ}$ of visual angle.

\section{Stimuli}

The sequence of screen events during a trial is given in Fig. 1. The background of the monitor remained nominally black $(R=G=B=0)$ during the experiment. Ensemble items and memory items were drawn in white.
Fixation To signal the imminent trial, a fixation mark was presented 100 pixels from the top of the screen and centered on the vertical midline. Its duration was $500 \mathrm{~ms}$ followed by a 250-ms blank screen prior to the memory items.

Memory items Memory strings contained 4-7 digits presented in DejaVu Sans Mono font at size 60 (each digit subtended $1.2^{\circ}$ horizontally and $1.3^{\circ}$ vertically). Half the observers saw memory strings of lengths 4 or 5 and the other half saw memory strings of lengths 6 or 7 . Within each length, half the strings were all zeros and the other half was randomly selected integers from an appropriate range of values. For example, to obtain a five-digit random number, the interval 10001 to 99999 was polled. Digits were presented as a contiguous string of appropriate length located 100 pixels from the top of the screen and centered left-to-right. The strings of lengths 4 or 5 were shown for $1.5 \mathrm{~s}$ and the strings of lengths 6 or 7 were presented for $2.1 \mathrm{~s}$ to allow approximately the same time per digit, for viewing.

Ensembles Ensembles contained nine horizontal lines whose lengths were drawn without replacement from the doubling of the set of lengths of 18 to 42 pixels in two-pixel increments. Thus a given size could occur a maximum of two times per ensemble and a minimum of zero times over the nine lengths. Positions of the nine horizontals were allocated to the nine cells of an imaginary $3 \times 3$ grid, which was centered on mid-screen. Cell centers were spaced 100 pixels from adjacent cells vertically or horizontally. The ensemble was drawn well below the location where the memory string had been and was present for a nominal $500 \mathrm{~ms}$.

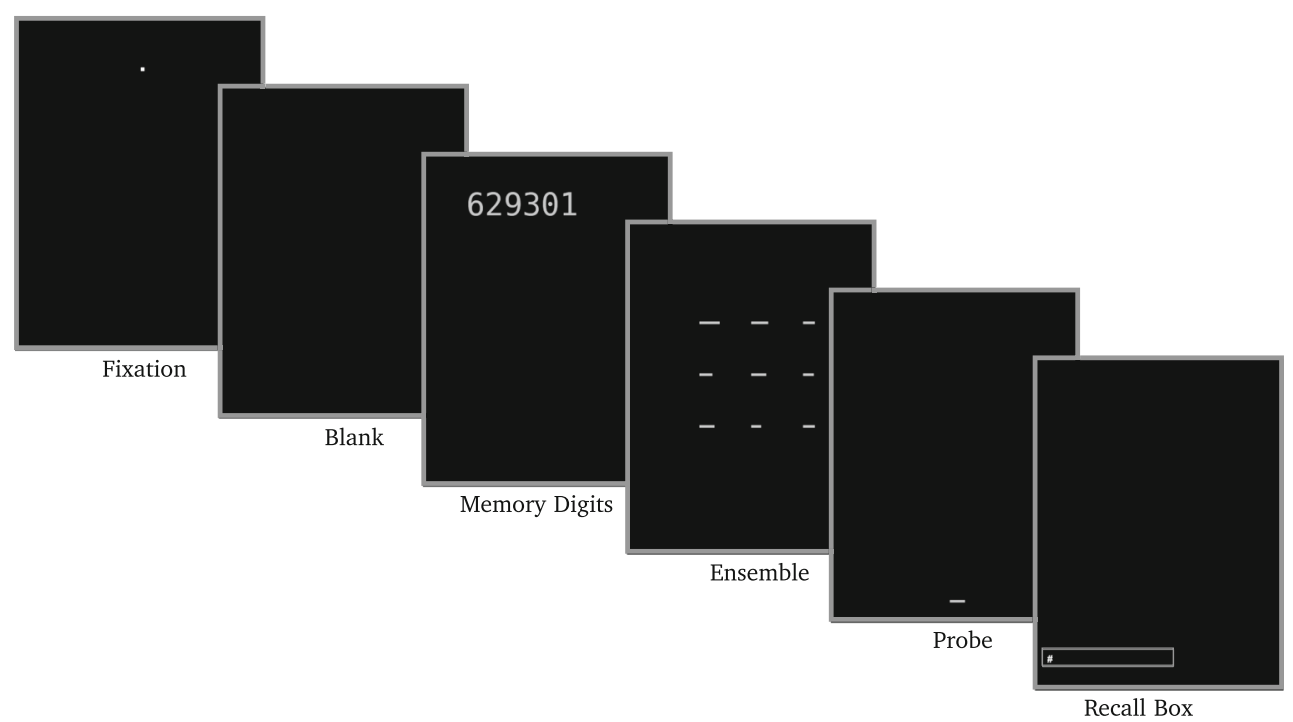

Fig. 1 These screen captures show only the central part of the display. The actual screen was in a 4:3 (W:H) aspect ratio $(36 \mathrm{~cm}$ wide by $27 \mathrm{~cm}$ high). For timings see text 
Probes On each trial, the integer value of the ensemble mean was computed and the probe length was generated according to a multiplier of $0.75,0.85,1.00,1.15$, or, 1.25 times the ensemble mean to create the five probe:ensemble mean RATIOs. Probe lengths were then converted to integers by truncation (flooring). The probe was presented well below the location where the ensemble had been and was offset to the left or to the right of the vertical midline by \pm 10 pixels equally often for each of the other conditions (RATIO, memory string length, and RANDs vs. ZEROs). A random (independent for each trial) vertical offset ranging from -15 to +15 pixels in three-pixel increments was also applied to the probe location. The probe was available until a response was made using the left ALT or right ALT keys on an Apple USB keyboard. Assignment of keys to "greater than" or "less than" was altered across observers in a haphazard fashion to approximately equate the two mappings.

Recall As soon as a response to the probe was recorded, a small rectangular entry box containing an octothorpe (\#) prompt was shown for observers to type in the memory item. Pressing the 'return' key logged the entered text and terminated the trial.

Each observer responded to 160 trials: five probe:mean RATIOs $\times 2$ left/right probe displacements $\times 2$ memory types (RANDs, ZEROs) $\times 2$ memory sizes $(4,5$ or 6,7 between groups) $\times 4$ replications. No feedback was given concerning accuracy on serial recall nor on responses to the probes.

\section{Procedure}

Observers were tested one at a time in a small darkened lab room. After signing the consent form, the program was started and the following instructions were displayed:

"This experiment investigates your ability to perform two tasks at once. Each task is relatively simple on its own, and I am trying to see if they can be combined with little added difficulty. On each trial, you will see a fixation + to alert you to get ready. Next comes a 4 or 5 digit number which you are to memorize, then a group of horizontal lines shown for about $1 / 2$ a second. After the group of lines is gone, a single line will appear and you need to press the RIGHT ALT/OPTION key if the single line is larger than the average or the LEFT ALT/OPTION key if the single line is smaller than the average of the sizes in the group of lines. You might think you are just guessing but many people are very good at this. After you have pressed the LEFT or RIGHT ALT/OPTION key, a box will appear for you to type in the number that you have memorized. Please type in as much as you remember and if you forget any digits, use an $\mathrm{x}$ or a dot as a placeholder. If the number is all zeros (0000 or 00000) you can just type a single 0.”
As appropriate, "4 or 5" was replaced with " 6 or 7" in the fourth sentence and the length of the zero strings was adjusted in the final sentence. The instructions for the key mapping were also changed for the reverse mapping.

\section{Results}

\section{Data screening: Rationale and implementation}

The primary indicator of the effect of memory load on averaging is the parameter set (especially slope) associated with the logistic psychophysical fit of $p$ ("probe is greater than mean") across the five RATIOs as a function of memory load type (RANDs vs. ZEROs). The steeper the ogive (larger slope), the smaller the size of the observer's interval of uncertainty (lower threshold) for the mean. Thus, if the slope of the probe judgment curve on RANDs trials is significantly higher than the slope for the ZEROs trials (and therefore the threshold is smaller for RANDs than ZEROs) in the context of acceptable levels of memory performance on the RANDs trials, there is evidence consistent with the hypothesis that dual tasking in this situation improves mean judgments.

The strength of this interpretation would then hinge on the quality of the two dependent measures, i.e., some appropriate level of recall performance as evidence that the observer was making a reasonable effort to retain and recall the numbers, and, stable parameter fits to the psychophysical curve based on sufficient data.

Three strategies are used to increase confidence in the data and analyses. First, a relatively large number of observers were tested in an effort to make criterion variables robust against noisy or outlier observations. A benefit of a large number of observers is that the estimated slope means will be more representative of the population value than if a smaller number of observers were tested. Second, an algorithmic (objective) observer rejection procedure was implemented to avoid contamination of the data by uncooperative observers and to reduce bias in the process (see below). Finally, the basic pattern of results will be demonstrated using several different types of analysis (and inclusion criteria) including ANOVA on the per-observer cell means, ANOVA on the fitted parameters, and judgments conditionalized on memory task performance, to show the systematicity of the findings.

For the first pass, responses on the DIGIT memory/recall task were inspected to verify that a reasonable effort had been made to retain and enter digits and placeholders as specified in the instructions. Data from two observers (of the original 191) were disqualified outright and are not included in any further analyses: one due to bogus responding (all RANDs trials except one had a response of " 1 " or "NA" on 
the recall task), and a second whose first 65 trials had "NA" recall response.

\section{ANOVAs with dependent measure $p$ ("probe is greater than mean")}

The probe judgments for the remaining 189 observers were then subjected to analysis based on the sensitivity of responding to non-unity probe:mean ratios. Of the total 160 trials per observer, there were 64 trials with the probe SMALLER than the ensemble arithmetic mean (RATIOs $0.75: 1$ and $0.85: 1$ ) and 64 trials with the probe LARGER than the ensemble arithmetic mean (RATIOs 1.15:1 and 1.25:1). See, Bauer (2009) for justification of the arithmetic mean in this case. Trials for these four ratios (i.e., excluding the $1: 1$ ratio trials) were sorted by response ("probe is larger than mean" or "probe is smaller than mean") to verify that for the RATIOs less than one, the dominant response was "probe is smaller than mean" and for the RATIOS greater than one, the dominant response was "probe is larger than mean".

Specifically, to retain the observer's data, counts in the two cells containing nominally correct responses, i.e., $p$ ("larger"|LARGER) and $p$ ("smaller"|SMALLER) needed to exceed the counts in the corresponding error cells, i.e., $p$ ("smaller"|LARGER) and $p$ ("larger"|SMALLER), respectively, by at least ten occurrences. Therefore, the number of correct trials needed to exceed 36 out of 64 and number of errors $<28$ out of 64 was required for both LARGER and SMALLER probes. The effect of this is to algorithmically reject observers who responded indiscriminately by randomly pressing the response keys or by using one response for the majority of trials. Thirty-seven observers' data failed this test, resulting in 152 remaining observers (76 in the 4,5 DIGIT group and 76 in the 6,7 DIGIT group). In actuality, this screening originally resulted in unequal numbers of observers in the two groups and the 6,7 DIGIT group was topped up to equate both groups at 76 observers.

ANOVAs and logistic curve fits were performed using the R-project version 3.2.0 functions "aov \{stats\}" and "glm \{stats\}" (https://www.r-project.org/). The ANOVA model included two within observer factors (probe:mean RATIO of $0.75,0.85,1.00,1.15$, and 1.25 as a numeric variable, and, two memory types RANDs vs. ZEROs as a categorical variable) and one between-groups factor of memory set size $(4,5$ or 6,7$)$ as a categorical variable. The dependent variable was $p$ ("probe is greater than mean"). For this analysis, only trials with perfect serial recall in the RAND condition and any number of zeros in the ZEROs condition were included. Analyses with other recall criteria are reported later.

The main effect of RATIO was significant, $F(1,150)=$ 4966, $p<.0001$, MSE $=0.03, \eta_{p}^{2}=0.970$. The memory set size effect $(4,5$ vs. 6,7$)$ was not significant $F<1$, $\eta_{p}^{2}=0.004$. The main effect of RANDs vs. ZEROs was not significant, $F(1,150)=2.63, p>.10), \eta_{p}^{2}=0.017$. The RANDs vs. ZEROs by RATIO interaction was significant $F(1,150)=19.3, p<.001, \eta_{p}^{2}=0.114$ (see Fig. 2). All other effects were $F<1$. Because memory set size did not exert a main effect or contribute to interactions, it will be dropped from the analyses below unless otherwise noted.

To verify the stability of the ANOVA results, a 10,000 replication randomization test was performed on the same data ignoring memory set size. In the randomization test, the ten observations of $p$ ("probe is greater than mean") per observer (5 RATIOs by 2 memory types) are randomly assigned i.e., disordered, (within observer and without replacement) to the ten cells. The analogy would be shuffling a deck of ten cards each with a $p$ ("probe greater") value for each observer and dealing them into the ten cells for that observer. This is done for all observers and the ANOVA is run in the usual way. The ANOVA F-statistics for the main effect of RATIO, the main effect of memory type, and the interaction of RATIO with memory type are then stored and the process is repeated for a number of replications (in the present case a total of 10,000). Following the final

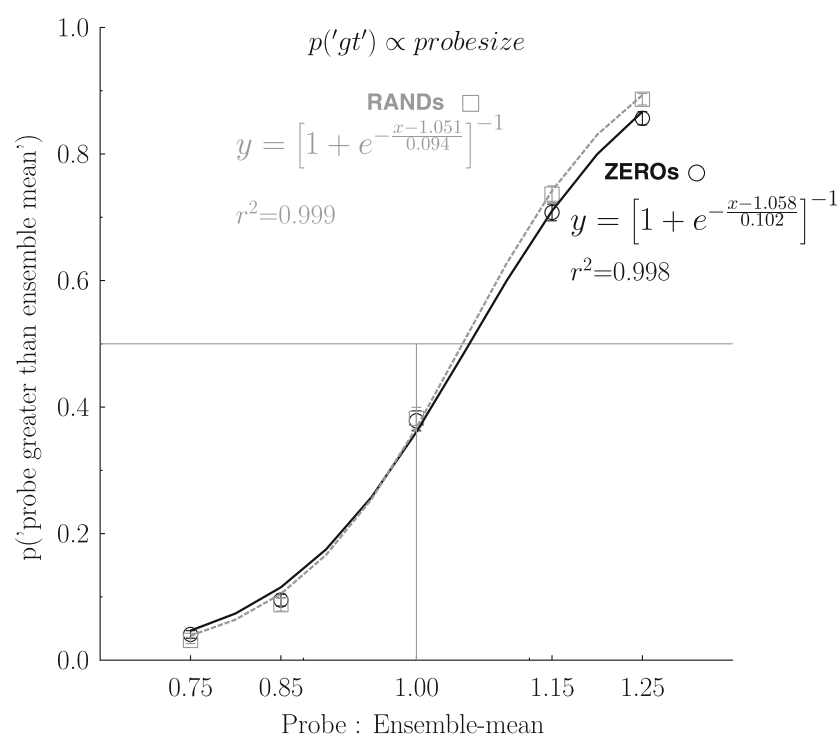

Fig. $2 p$ ("probe is greater than mean") as a function of RATIO and memory type ZEROs (circle markers) versus RANDs (square markers). Standard error bars $( \pm 1)$ are about the size of the plotting symbols. The curves are the best-fit logistic functions (ZEROs solid line, RANDs dashed line). In this form, $y=\left[1+e^{-\frac{x-\alpha}{\beta}}\right]^{-1}$, the logistic fit yields $\alpha$, which is the PSE (point of subjective equality) and the standard deviation $\beta$ (inverse slope). There is a 5.1 and $5.8 \%$ overestimate of the ensemble mean for RANDs and ZEROs, respectively, and greater precision (smaller standard deviation (0.094 vs. 0.102) for RANDs versus ZEROs, respectively). Corresponding logistic slopes are 10.67 for the RANDs and 9.78 for the ZEROs. Note that the logistic fits here are the simple curve fits to the two sets of five data points in the graph. Observer-level fits are discussed below 
repeat, the proportion of cases in the randomizations with the $\mathrm{F}$ (for each main effect and the interaction) exceeding the Fs from the ordered ANOVA with intact conditionto-response correspondence is computed. The question answered is: how frequently would an F-statistic as large as the one from the ordered ANOVA occur in the randomizations of the observations to cells. During 10,000 randomizations, $p(F>4973)$ for the main effect of RATIO and $p(F>19.4)$ for RATIO by memory type (RAND vs. ZERO) were both 0 , and for the main effect of memory type $p(F>2.63)=0.107$. (The $F$ values here differ slightly from those in the main ANOVA above due to the change in degrees of freedom associated with dropping the memory size factor.)

In the analyses above, perfect serial recall was required for a given trial to contribute to the ("probe greater") tally. An additional set of analyses was performed with a relaxation of the memory scoring criteria. In these analyses, the retained 152 observers' data were screened trial-by-trial according to the proportion of individual digits in the correct serial position on the RANDs trials. For ZEROs trials the typed in value had to be any number of zeros. Then ANOVAs based on the model above (excluding memory size) were run including only trials with the proportion of correctly positioned digits greater than or equal to 0.0 to 1.0 in increments of 0.05 . At 0.0 , all trials were included and at 1.0 the analysis is the same as for perfect serial recall above. The $F$ value for the interaction of RATIO by memory type (RANDs vs. ZEROs) was significant in all cases and was proportional to the memory inclusion criterion, $r^{2}=0.89$, $p<.001$. The same was true of the $\eta_{p}^{2}$ values, $r^{2}=0.89$, $p<.001$.

Section summary: In both a standard ANOVA and in the randomization test, the interaction of RATIO with memory type (RANDs vs. ZEROs) was significant. The effect size is modest but it does reveal improvement in the mean-size judgments as realized in the higher slope for the RANDs vs. ZEROs curve. Further, the effect size was related to the strictness of the serial recall scoring procedure, i.e., the better the digit memory, the larger the interaction effect size. An additional result of interest is that the 50:50 point on the $y$-axis (the point at which observers supposedly cannot tell the difference between the probe size and the ensemble mean) does not correspond to a probe:mean ratio of 1.00 on the $x$-axis. Thus the point of subjective equality (PSE) is not as would be expected if observers were perfect perceivers of the probe line and the ensemble mean. PSEs can be read from the alpha $(\alpha)$ parameter in logistic fits in Fig. 1. What is important is that whatever is driving this bias appears to be independent of RANDs vs. ZEROs; the PSE for RANDs (1.051) is numerically smaller than the PSE for ZEROs (1.058). This will be corroborated using observer-level fits to test the difference statistically.

\section{Observer-level logistic fits}

\section{Logistic slopes}

Logistic curve fits per observer were performed using the R-project version 3.2.0 function "glm \{stats\}" (https://www. r-project.org/). Descriptive statistics on all 304 slopes (two per observer; RANDs and ZEROs ignoring memory size) showed a mean of 15.13 ( $\mathrm{SD}=13.99)$, a median of 12.39 , and a skew of 5.23. This indicates a large right tail in the distribution suggesting the possible presence of outliers. In fact, the highest slope value in the ZEROs condition was 33.3 and in the RANDs condition, two slopes exceeded 120. First, analysis of raw slopes is presented, then the analysis of these slopes with techniques to reduce the effect of the large slopes are discussed.

Larger slopes were associated with the RANDs condition $(\mathrm{M}=17.79, \mathrm{SD}=18.79)$ compared with the ZEROs condition $(\mathrm{M}=12.47, \mathrm{SD}=5.06)$ and this difference was significant, $F(1,151)=12.22, p<.001, \mathrm{MSE}=175.72, \eta_{p}^{2}=0.074$.

The present concern, however, is typical performance for both RANDs and ZEROs judgments. This creates a dilemma in how to deal with any overly influential values without manufacturing a spurious difference between groups (or erroneously creating a null effect). One strategy is to log-transform the slopes prior to the ANOVA. Using natural logarithms, the slope of the RANDs condition is significantly larger than the slope of the ZEROs condition, $F(1,151)=20.27, p<.001$, MSE $=0.1573$. A second method to further verify that this significant difference was not the result of some artifactual extrema is to screen the slopes with a commonly used outlier identification procedure, viz, (Van Selst \& Jolicoeur, 1994), which has been cited over 400 times in Web of Science (as of Sept 2015). Briefly, for a given data cell, the mean and standard deviation are calculated omitting the largest and smallest values. If either of these values exceeds the mean by \pm 4 standard deviations, it is eliminated, the new mean and standard deviation are computed and the new largest and smallest values are tested against the \pm 4 standard deviation criteria. This process iterates until no additional values are eliminated.

For this analysis, the Van Selst and Jolicoeur (1994) algorithm was applied to the four cells of the crossing of memory set size $(4,5$ vs. 6,7$)$ with RANDs vs. ZEROs. This flagged 12 slopes as outliers: three observations in the 4,5 data, and nine observations in the 6,7 data. Because RANDs and ZEROs was a within-observers measure (each observer provided one slope in the RANDs condition and a corresponding slope in the ZEROs condition), both observations for a given observer were removed if the slope in either the RANDs or ZEROs condition was declared an outlier. This has the effect of creating unequal Ns across the two 
memory sizes 6,7 (67 observers) and 4,5 (73 observers), but still permits the ANOVA with RANDs and ZEROs as a within-observer factor and the two memory sizes as a between-groups factor (removing only the offending value from RANDs or ZEROs only would have necessitated a less appropriate independent $t$ tests even though most of the data were paired). The main effect of RANDs vs. ZEROs on slope was significant $F(1,138)=8.3, p<.005$, $\mathrm{MSE}=14.644, \eta_{p}^{2}=0.056$ (see Fig. 3). The main effect of memory set size and its interaction with RANDs vs. ZEROs were both Fs $<1$.

\section{Analysis of serial recall performance and effect on logistic slopes}

Across the 12,160 RANDs trials ( 152 observers $\times 80$ trials), 9651 had perfect serial recall (.793). A further 1888 had the correct number of digits or placeholders and generally had transposed digits but were otherwise similar to the target number. Of the 621 remaining errors, 83 had no digits or a 0 entered and the rest were close to the target number but had the placeholder ' $\mathrm{X}$ ' for a forgotten digit as was instructed, too many, too few, and misordered digits.

For the 12,160 ZEROs trials, 11,901 had correct recall (.978) of which 9054 were entered as a single zero, and 2847 were entered as the number of zeros indicated at the beginning of each trial. The remaining 259 cases consisted primarily of no response entered and perseverations (digits from immediately preceding trial entered).

Using strict scoring (perfect serial recall in RANDs trials and any number of 0s entered in ZEROs trials), an ANOVA with proportion of error as the dependent measure and the independent variables of two memory types (RANDs, ZEROs) and five probe:mean RATIOs revealed

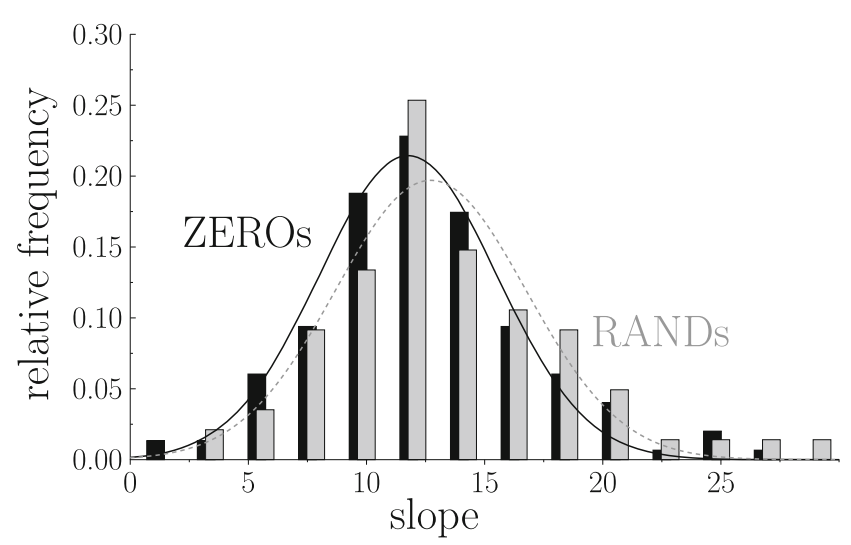

Fig. 3 Relative frequency histograms for slopes from the logistic fits of the probe judgment task for ZEROs (black bars and solid line) versus RANDs (gray bars and dashed line) memory load conditions. These slopes are from the trimmed data set using the Van Selst and Jolicoeur (1994) algorithm. The normal fits produced a mean of 12.7 for the RANDs and 11.7 for the ZEROs the following. There was a main effect of memory type, $F(1,151)=126.0, p<.0001, \mathrm{MSE}=0.103, \eta_{p}^{2}=0.455$, and a main effect of RATIOs, $F(1,151)=3.91, p<.05$, $\mathrm{MSE}=0.004, \eta_{p}^{2}=0.025$

The interaction was also significant, $F(1,151)=7.06$, $p<.01, \mathrm{MSE}=0.509, \eta_{p}^{2}=.045$. Proportion error for this analysis is shown in Fig. 4. Proportion error ranged from 0.017 to 0.027 for the ZEROs condition and 0.188 to 0.216 in the RANDs condition. A reviewer noted that the psychophysical fits for the RANDs condition were therefore based on fewer trials than those for the ZEROs trials because fewer of the RANDs trials were associated with correct free recall. The concern is that the logistic fits might therefore be biased by this difference. One way to investigate this is to take the observer-level data at each RATIO and when the number of trials excluded for serial recall errors the RANDs trials exceeds the exclusions in the ZEROs trials, randomly omit the appropriate number of ZEROs trials to balance the two. Then recalculate the p ("greater than") score based on the reduced number of observations and refit the curves to obtain new slopes. Selection of which trials to drop was performed randomly, and 1000 resamplings were performed. The outcome of this procedure is shown in Fig. 5. With the number of observations thus balanced, the majority of slope differences (slopeRANDs-slopeZEROs) are larger than 0 .

\section{Logistic PSE}

Logistic fits also produced the point of subjective equality (PSE). The mean PSEs (1.0517 for RANDs and 1.0598

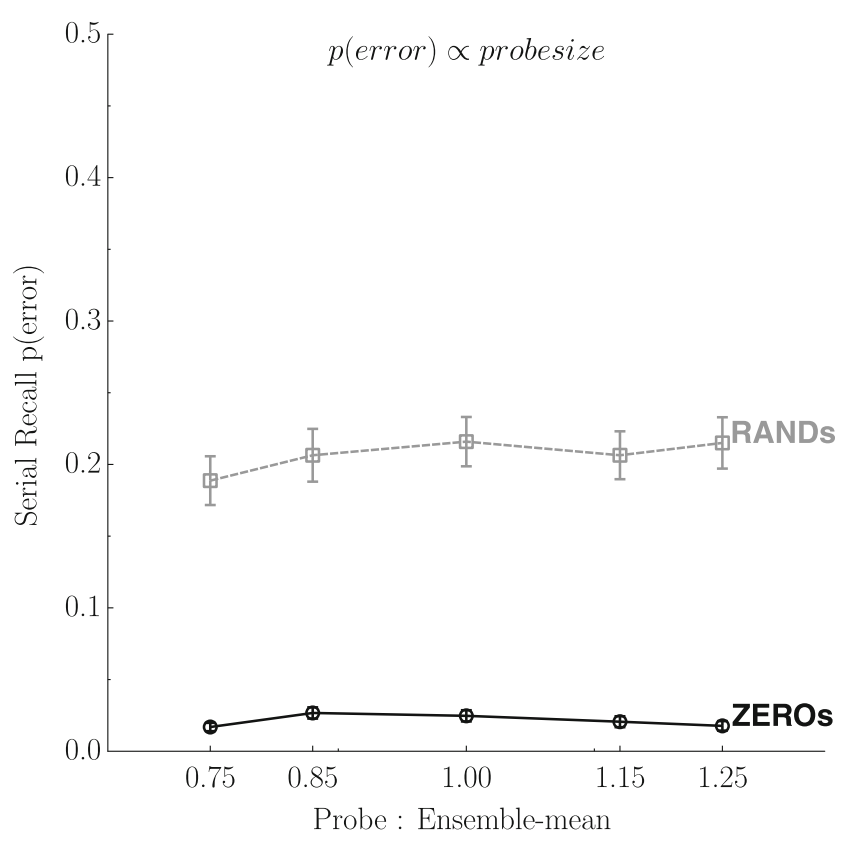

Fig. 4 Proportion error in serial recall as a function of memory item type (ZEROs, solid black line; RANDs, dashed gray line) 


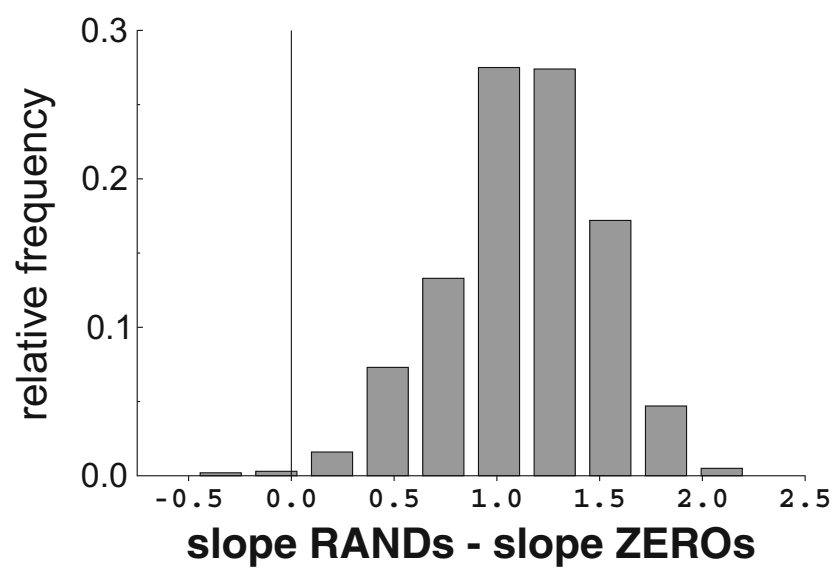

Fig. 5 Relative frequency histogram of slope difference (RANDsZEROs) with number of observations balanced across RANDs and ZEROs (see text). The thin vertical line indicates no slope difference. The mean slope difference is 1.18

for ZEROs) were almost identical to those computed on the aggregated data in Fig. 2. The difference based on these observer-level fits approached significance, $F(1,151)=$ $3.304, p<0.072$, MSE $=0.0019, \eta_{p}^{2}=0.0214$. This difference of less than $1 \%$ (similarly $1 \%$ in the medians) is quite small and will be ignored except to note that there appears to be no greater bias in the RANDs vs. ZEROs conditions so the slope advantage in the RANDs condition can be interpreted without concerns for differential bias.

\section{The PSE shift}

The PSEs were uniformly shifted to about +5 to $+46 \%$, suggesting overestimation of the ensemble mean (or underestimation of the probe size). In Bauer (2009), there was also an overestimation of about $2.5 \%$ for ensembles of 16 horizontal lines using a method of adjustment procedure. Chong and Treisman (2003, Appendix A) report overestimation of arithmetic means by about $8 \%$ (inferred from their Fig. 6) in a method of adjustment procedure using ensembles of two lines. Thus, some rightward shift of the PSEs in the present experiment is not unprecedented. Whether some of this bias is a systematic property of the averaging process will require further experimentation. It is enough to note that this PSE bias was equivalent in the RANDs vs. ZEROs conditions (Fig. 6).

Interim summary Logistic fits indicated that a memory load of RANDs was associated with a steeper slope (smaller standard deviation) when compared with the ZEROs condition and no difference in PSE. The PSEs were uniformly shifted to about $5-6 \%$ overestimation of the ensemble mean (or underestimation of the probe size). This indicates better averaging performance with the load of RANDs than with the ZEROs with no concomitant difference in the bias. The

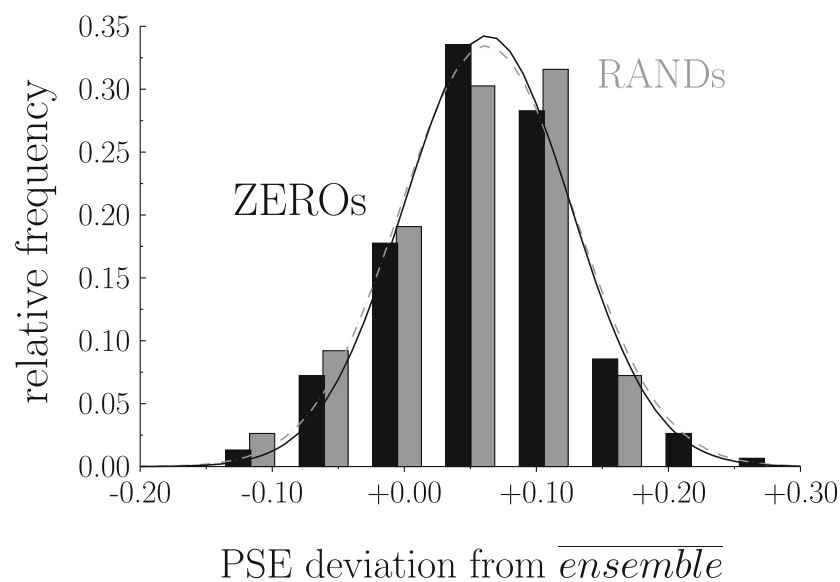

Fig. 6 Relative frequency histograms for PSE deviation for ensemble means for ZEROs (black bars and solid line) versus RANDs (gray bars and dashed line) memory load conditions. Both mean PSEs deviated from the expected value of 0.0 . The normal fits produced a mean of +0.062 for the RANDs and +0.062 for the ZEROs

slope benefit of RANDs over ZEROs maintains when number of trials contributing to the slope fit are balanced across RANDs and ZEROs (see text for balancing algorithm).

\section{Response time results}

The instructions to the observers did not contain any suggestion of how to allot time or effort to the two tasks on each trial. The question arises as to how they might have strategically (or otherwise) managed the demands of the probe task to harmonize with the requirements of memory task as a function of the RANDs trials versus the ZEROs trials. On each trial of the experiment, the elapsed time from the probe onset to judgment keypress was logged. These reaction times (RTs) can be examined for insights into why the RANDs memory condition was generally associated with better psychophysical performance on the averaging task than the ZEROs condition.

The following analysis includes data from the 152 observers who passed the two screening criteria mentioned at the beginning of the Results section. Only probe reaction times from trials with a correct serial recall are included. For this analysis, the memory size factor $(4,5$ or 6,7$)$ was reintroduced. Pre-screening of the RTs for outliers using the Van Selst and Jolicoeur (1994) procedure rejected 1022 of 21,549 reaction times (4.74\%). The remaining probe RTs were analyzed with an ANOVA model that included two within- groups factors (five probe:mean RATIOs and two memory types, i.e., RANDs/ZEROs) and one betweengroups factor of memory size $(4,5$ or 6,7$)$.

There was a main effect of RANDs $(\mathrm{M}=1495 \mathrm{~ms})$ versus ZEROs $(\mathrm{M}=1619 \mathrm{~ms}), F(1,150)=36.9, p<.001$, $\mathrm{MSE}=159227, \eta_{p}^{2}=0.197$ and of RATIO, $F(1,150)=$ $40.8, p<.001, \mathrm{MSE}=217453, \eta_{p}^{2}=0.226$. The main effect 
of memory set size $(4,5 \mathrm{M}=1495 \mathrm{~ms} ; 6,7 \mathrm{M}=1620 \mathrm{~ms})$ was $F(1,150)=3.72, p<.06, \mathrm{MSE}=1621052, \eta_{p}^{2}=$ 0.024 . The only significant interaction was between RATIO and memory type (RANDs vs. ZEROs), $F(1,150)=7.26$, $p<.01, \mathrm{MSE}=78136, \eta_{p}^{2}=0.046$. All other $F \mathrm{~s}<1.40$.

Figure 7 shows the mean RT for both memory size groups and RANDs vs. ZEROs across RATIOs. Probe responses on trials with RANDs (dashed lines with '\#' plotting symbol) were faster than on trials with ZEROs (solid lines with ' 0 ' plotting symbol) for both memory sizes. Given that memory size and RANDs vs. ZEROs are contextual variables (i.e., the digits precede, and the response to them follows the psychophysical task), it is noteworthy that they exert such a large effect in probe judgment time.

Both RANDs curves (dashed gray lines) are below their corresponding ZEROs curves (solid black lines). This suggests that observers were pushing themselves to faster probe responses (or deliberating less) when the memory task was more vulnerable to error (i.e., on RANDs trials because on ZEROs trials, a single 0 was sufficient as a response). It may be that observers had some insight concerning their approximate running success on the memory task. Recognizing whether they had remembered enough digits or at least how fluently the digits came to mind on recent recalls could help form this insight. On RANDs trials, there was a negative correlation between serial recall score (correct vs. incorrect) and RT on the averaging task even with memory length accounted for, $r=-0.083, t(12144)=9.94, p<.001$.

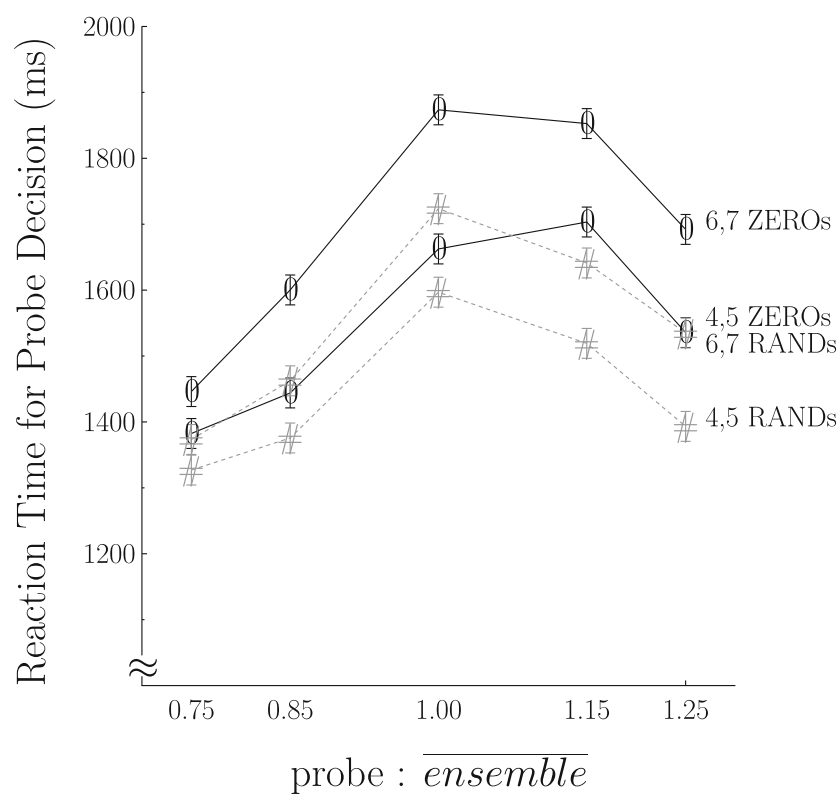

Fig. 7 Reaction time for probe decisions for ZEROs (solid lines, '0' plotting symbol) versus RANDs (dashed gray lines, '\#', plotting symbol) memory load conditions. Within-observer standard error bars were computed according to Loftus and Masson (1994) as $\sqrt{\frac{78136}{152}}$ where 78,136 is the MSE for the RATIO $\times$ RANDs vs. ZEROs interaction and 152 is the number of observers
The probe response was far more subjective and offered little to help assess success or failure. Therefore, if serial recall performance started to slip, they could push the probe judgment time down to increase accuracy on recall.

If this is true and observers use the probe judgment task elastically, then there is a concern that rushed probe judgments might be less accurate. Indirectly, there is evidence against this in the sense that faster judgments are associated with the RANDs condition, which also produced psychophysical functions with steeper slopes. Another way to look at this would be to take the RTs for RANDs trials and sort them according to whether the probe decision was 'correct' or 'incorrect' for that trial.

Determination of correctness was based on the assumption that observers compute the arithmetic mean of the ensemble lengths and compare this mean with the length of the probe. A further assumption is that once computed, the ensemble mean endures long enough and without systematic memory effects until the probe judgment is made. The magnitude of the ensemble mean in memory is assumed not to alter the perception of the probe systematically. This is the ideal observer. Because there is no correct answer for the $1: 1$ RATIO, this condition is not considered in this analysis. Given the shapes of the ogives in Fig. 2, the number of trials with probe responses considered 'incorrect' will be small. Median RT, and, mean RT following outlier screening according to Van Selst and Jolicoeur (1994) default method, are given in Table 1 as a function of RATIO and correctness. For each RATIO, RT for 'correct' judgments is faster than for 'incorrect' judgments.

Interim summary Memory load of random digits was associated with increased speed of responding to the probe and higher normative accuracy. The 6,7 digit memory size condition adds about $100 \mathrm{~ms}$ per trial to the probe judgment time

Table 1 Median and screened mean RT for correct and incorrect judgments for RATIOs $0.75,0.85,1.15,1.25$

\begin{tabular}{lllll}
\hline $\begin{array}{l}\text { Probe } \\
\text { Judgment }\end{array}$ & RATIO & $\begin{array}{l}\text { Median } \\
\text { RT }(\mathrm{ms})\end{array}$ & $\begin{array}{l}\text { Mean } \dagger \\
\text { RT }(\mathrm{ms})\end{array}$ & $\begin{array}{l}\text { number of } \\
\text { observations }\end{array}$ \\
\hline error & 0.75 & 1483 & $1566 \mathrm{SD}=601$ & 59 \\
correct & 0.75 & 1212 & $1273 \mathrm{SD}=474$ & 1914 \\
error & 0.85 & 1432 & $1686 \mathrm{SD}=839$ & 164 \\
correct & 0.85 & 1231 & $1313 \mathrm{SD}=500$ & 1766 \\
error & 1.15 & 1683 & $1798 \mathrm{SD}=850$ & 515 \\
correct & 1.15 & 1352 & $1432 \mathrm{SD}=551$ & 1415 \\
error & 1.25 & 1833 & $2098 \mathrm{SD}=1131$ & 217 \\
correct & 1.25 & 1281 & $1358 \mathrm{SD}=494$ & 1693 \\
\hline
\end{tabular}

$\dagger$ screened using Van Selst and Jolicoeur (1994) method. Therefore, the number of observations in mean RT is $3-7 \%$ smaller than shown in rightmost column 
over the 4,5 memory size but does not change the benefit of low vs. high load on the logistic slopes. Therefore, the 4,5 RANDs digit condition (fastest over all on probe judgments) seems more efficient experimentally than the 6,7 condition.

\section{Memory load and the number of observers needed}

A relatively large number of observers were tested in each of the 4,5 and 6,7 DIGIT memory conditions. The choice of 4-7 DIGITs was in line with other research using DIGITS as memory load (see, for example, Aldred, Crawford, Duffy, \& Smith, 2016; Rissman, Gazzaley, \& D'Esposito, 2009) and was intended to be close to memory span on average. That memory load size did not seem to be important in the present experiment suggests that the slope benefit of the RANDs vs. ZEROs condition could be obtained with just the 4,5 DIGIT load which observers might find less frustrating than the 6,7 DIGIT load. This would also result in retention of more trials in an analysis with strict serial order recall requirements. In fact, given the existence of significant individual differences in digit span and other verbal memory span measures, it might be prudent to pretest each observer's digit span and set the number of digits in the memory task accordingly (e.g., Cocchini et al., 2002). It is also possible that even though the ZEROs memory load was intended to be trivial, just having to remember that a given trial was a ZERO trial may still impose a non-trivial load on the observer. If this is true, then the present experiment may underestimate the memory load effect difference that would obtain if the ZERO trials had been replaced with no memory requirement at all as a control condition. In fact, on 2585 trials or about $21 \%$ of the 12,160 ZEROs trials ( 80 each for 152 observers), the number of zeros typed in by the observer was the same as the number of zeros shown at the beginning of the trial. Thus on these trials, the observers were memorizing more than needed and the memory load was not as low as intended.

Steeper slopes and therefore smaller threshold values for perceptual averaging of lines resulted from the RANDs versus ZEROs memory load. One of the implications of this is that any critical tests of the influence of other factors (e.g., number of items to average, changes in variability of the members, exposure time, focused versus diffuse attention) on averaging efficiency could benefit from including a load condition to determine whether the effects of these other factors are the same when thresholding is more accurate. A natural question is how many observers would be required to demonstrate a differential load effect. To provide some evidence to answer this question, data from the 152 observers in the present experiment were reanalyzed in groups of 10 to 100 observers in steps of five observers. For each of these 19 sampling sizes, 1000 replications were analyzed with the appropriate number of observers drawn randomly and without replacement from the pool of 152. Each replication was analyzed with an ANOVA using p ("greater than") as the dependent variable with RATIO and RANDs vs. ZEROs as independent variables under the strict serial recall criterion. The interaction of RATIO with RANDs vs. ZEROs was the indicator of slope difference so the $p$ value for each replication on this interaction was tallied as a function of sampling size. Of course, the significance of the interaction is not the sole figure of merit here: the interaction must be in the correct direction as well. In only a single case during 19,000 replications was significance (i.e., $p<.05$ ) obtained for the slope of ZEROs greater than RANDs. This occurred for a sampling size of 10 . All other replications with the slope of ZEROs greater than RANDs were $p>.05$. The uppermost bars (dark solid [black]) in Fig. 8 show the percentage of non-significant $(p>.05)$ cases in which the slope of the ZEROs data was greater than the slope of the RANDs data, that is, numerically reversed from the analyses above. Of main interest are the other three bar types. For example, for number of observers $=10$, the bottom most bar (solid light [green if viewing in color]) shows that about $5 \%$ of $p<.01$ cases occurred (that is a significant RATIO $\times$ RANDs vs. ZEROs interaction in the expected direction) and the next bar upwards (diagonal hash [cyan if viewing in color]) indicates that about $17 \%$ of cases had

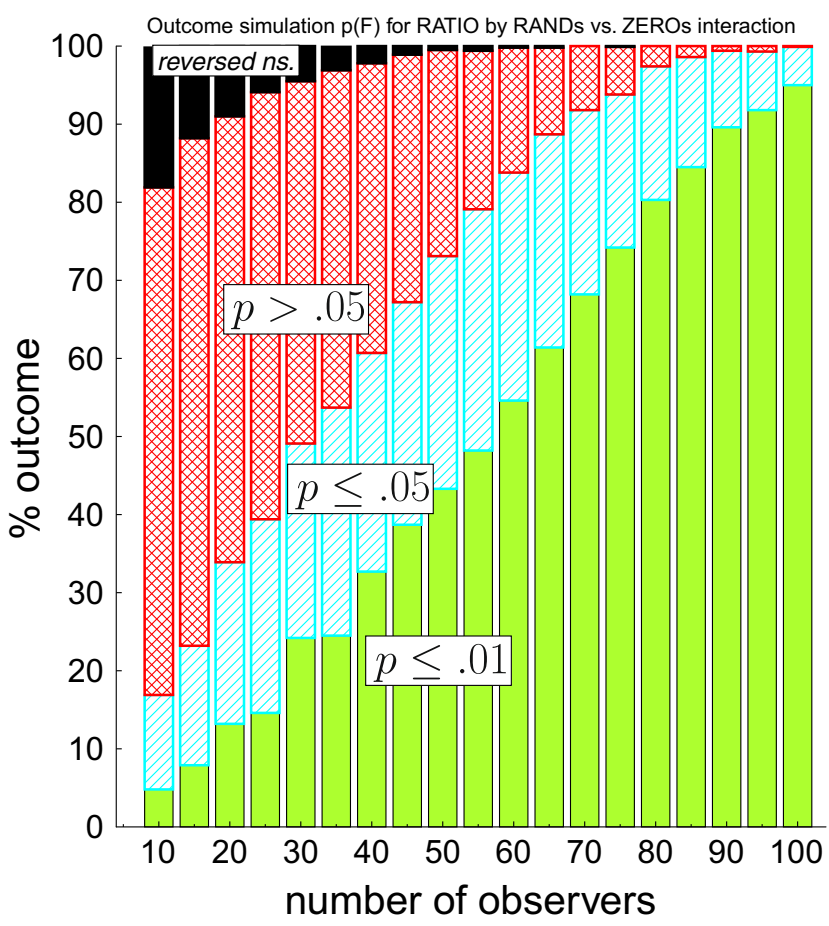

Fig. 8 Percentage of $p$ values for the RATIO by RANDs vs. ZEROs interaction as a function of sampling size. Top (dark bars) indicate nonsignificant reverse effect. Crosshatched bars show percentage nonsignificant effects in the expected direction (slope of RANDs larger than slope of ZEROs). Cases with $p<=.05$ include the right hash and light solid bars (cyan and green if viewing in color). Cases with $p<=.01$ are the light solid bars (green if viewing in color) 
$p<.05$, which includes the $p<.01$ cases. Thus, the combined height of the bottom two bars indicates the number of cases with $p<.05$. The next bar upwards (cross hatch [red if viewing in color]) indicates the number of cases with the slopes in the expected direction, but that failed to reach a criterion of at most, $p<.05$.

What this plot shows is that, for example, the probability of a significant (at $p<.05$ ) RATIO $\times$ RANDs vs. ZEROs interaction in the expected direction is about $50 \%$ with sample sizes in the mid thirties or, for $p<.01$, about double the number of observers. Note that the observers were randomly selected from the two memory set size groups. This same analysis was repeated excluding the same 12 observers flagged as outliers in the unequal $\mathrm{N}$ slope analysis above. The results were similar but as would be expected, the number of observers needed for a given level of significance increased (i.e., whereas a $50 \%$ chance of significance at $p<.05$ required about 32 observers as shown above with all 152 observers, the reduced set of 140 observers would require about 40 observers for the $p<.05$ level).

Even if the goal of a given experiment does not require demonstrating a significant RANDs vs. ZEROs effect, the present experiment still suggests that slopes will, on average, be greater with a near-span memory load than with minimal memory load. Of the 152 observers, there were 96 (63\%) with slopes in the RANDs condition larger than in the ZEROs condition.

\section{General discussion}

The practical conclusion from the present experiment is that introducing the memory load of RANDs was sufficient to increase the slope of the $p$ ("probe is greater than mean") by RATIO curves demonstrating finer mean size estimation than in the ZEROs condition. Probe responses were also faster and more accurate under the RANDs load than with the ZEROs load, which implies that 'snap judgments' are better. If similar threshold sharpening could be found by simply adding a probe decision deadline (i.e., removing the memory task completely and using the Response Signal Procedure such as in McElree \& Dosher, 1989), such a procedure might be preferred provided it did not frustrate the observers and the time-per-trial were reduced compared with holding a memory load. This might also permit more trials per condition for a given observer or session length, or more observers per hour. Again, rushing the decisions with the deadline would need to be tested to discover if it is the speeding per se, or the memory loading proper, which conveys the benefit.

It would be interesting to see if a slightly different verbal memory task requirement alters the benefit of high load over low load. For example, Cocchini et al. (2002) found little interference between a verbal memory task and visuo-spatial task supposedly because the verbal memory task is thought to draw little on visuo-spatial processing. However, with modification of the memory task (i.e., requiring vocal report of the memory digits in addition to typing them), Morey and Cowan (2005) found (negative) interference of the verbal task on a visual change detection task.

As it stands, it is unclear whether there were any implications for the averaging performance, of the visual characteristics of the memory items as presented (e.g., spatial frequency, character width) or the perceptual process changes in going from symbolic (numerals) to ensemble-wide featural (length) processing. In other words, was any of the threshold reduction and speed up benefit a fortuitous side effect of visual components serial recall task, and if so, what might that suggest about how the perceptual averaging process works? Such effects seem unlikely, but given the proposed ubiquity of the averaging process mentioned in the introduction, they cannot be ruled out for the present experiment. Perhaps auditory rather than visual presentation of the memory items would resolve this.

Finally, there is some evidence (e.g., in adults: Solomon, Morgan, \& Chubb, 2011; and in infants: Tuerk, 2014) that pooling and averaging of size information is in some way different from the same processes for orientation. Thus, replication of the present effect with other stimulus dimensions is necessary. Success or failure of the effect of load on other dimensions would be interesting and might provide clues concerning the averaging process and how basic stimulus dimensions are processed.

Acknowledgements Some of the results contained herein were presented at the 24th Annual Meeting of the Canadian Society for Brain, Behaviour and Cognitive Science, 2014. The author notes the contribution of Geoffrey Laforge in collecting some of the data, and thanks Jennifer Corbett and three anonymous reviewers for their helpful guidance and suggestions.

\section{References}

Aldred, S. R., Crawford, L. E., Duffy, S., \& Smith, J. (2016). Working memory and spatial judgments: Cognitive load increases the central tendency bias. Psychonomic Bulletin Review, 2016 Apr 15. [Epub ahead of print]. doi:10.3758/s13423-016-1039-0

Algom, D., Wolf, Y., \& Bergman, B. (1985). Integration of stimulus dimensions in perception and memory: Compositional rules and psychophysical relations. Journal of Experimental Psychology: Human Perception and Performance, 114(4), 451-471. doi:10.1037/0096-3445.114.4.451

Allik, J., Toom, M., Raidvee, A., Averin, K., \& Kreegipuu, K. (2013). An almost general theory of mean size perception. Vision Research, 83, 25-39. doi:10.1016/j.visres.2013.02.018

Allik, J., Toom, M., Raidvee, A., Averin, K., \& Kreegipuu, K. (2014). Obligatory averaging in mean size perception. Vision Research, 101, 34-40. doi:10.1016/j.visres.2014.05.003 
Alvarez, G. A. (2011). Representing multiple objects as an ensemble enhances visual cognition. Trends in Cognitive Sciences, 15(3), 122-131. doi:10.1016/j.tics.2011.01.003

Alvarez, G. A., \& Oliva, A. (2008). The representation of simple ensemble features outside the focus of attention. Psychological Science, 19(4), 392-398. doi:10.1111/j.1467-9280.2008.02098.x

Alvarez, G. A., \& Oliva, A. (2009). Spatial ensemble statistics are efficient codes that can be represented with reduced attention. Proceedings of the National Academy of Sciences, 106(18), 73457350. doi:10.1073/pnas.0808981106

Anderson, B., \& Druker, M. (2013). Attention improves perceptual quality. Psychonomic Bulletin \& Review, 20(1), 120-127. doi:10.3758/s13423-012-0323-x

Anderson, N. H. (1964). Test of a model for number-averaging behavior. Psychonomic Science, 1, 191-192. doi:10.3758/BF03342858

Ashourian, P. (2011). Bayesian inference underlies the contraction bias in delayed comparison tasks. PLoS ONE, 6(5), e19551. doi:10.1371/journal.pone.0019551x

Attneave, F. (1954). Some informational aspects of visual perception. Psychological Review, 61(3), 183-193. doi:10.1037/h0054663

Baijal, S., Nakatani, C., vanLeeuwen, C., \& Srinivasan, N. (2013). Processing statistics: An examination of focused and distributed attention using ERP. Vision Research, 85, 20-25. doi:10.1016/j.visres.2012.09.018

Barlow, H. (2001). Redundancy reduction revisited. Network: Computation in Neural Systems, 12(3), 241-253. doi:10.1080/net.12.3.241.253

Bauer, B. (2009). The danger of trial-by-trial knowledge of results in perceptual averaging studies. Attention, Perception, \& Psychophysics, 71(3), 655-665. doi:10.3758/APP.71.3.655.x

Bauer, B. (2015). A selective summary of visual averaging research and issues up to 2000. Journal of Vision, 15(4). doi:10.1167/15.4.14

Bocanegra, B. R., \& Hommel, B. (2014). When cognitive control is not adaptive. Psychological Science, 25(6), 1249-1255. doi:10.1177/0956797614528522

Chong, S. C., \& Treisman, A. (2003). Representation of statistical properties. Vision Research, 43(4), 393-404. doi:10.1016/S0042-6989(02)00596-5

Chong, S. C., \& Treisman, A. (2005a). Attentional spread in the statistical processing of visual displays. Perception \& Psychophysics, 67(1), 1-13. doi:10.3758/BF03195009

Chong, S. C., \& Treisman, A. (2005b). Statistical processing: Computing the average size in perceptual groupings. Vision Research, 45(7), 891-900. doi:10.1016/j.visres.2004.10.004

Cocchi, L., Toepel, U., De-Lucia, M., Martuzzi, R., Wood, S. J., Carter, O., \& Murray, M. M. (2011). Working memory load improves early stages of independent visual processing. Neuropsychologia, 49, 92-102. doi:10.1016/j.neuropsychologia.2010.10.021

Cocchini, G., Logie, R. H., Della Salla, S., MacPherson, S., \& Baddeley, A. (2002). Concurrent performance of two memory tasks: Evidence for domain-specific working memory systems. Memory \& Cognition, 30x(7), 1086-1095. doi:10.3758/BF03194326

Corbett, J. E., \& Melcher, D. (2014). Characterizing ensemble statistics: mean size is represented across multiple frames of reference. Attention, Perception \& Psychophysics, 76(3), 746-58. doi:10.3758/s13414-013-0595-x

Dakin, S. (2014). Seeing statistical regularities: Texture and pattern perception. In Wagemans, J. (Ed.), Oxford Handbook of Perceptual Organization (in press). Oxford: Oxford University Press. http://gestaltrevision.be/en/our-publications/handbook-of-perceptualorganization/accepted-chapters

Dakin, S. C., Bex, P. J., Cass, J. R., \& Watt, R. J. (2009). Dissociable effects of attention and crowding on orientation averaging. Journal of Vision, 9(11), 28. doi:10.1167/9.11.28
Dubé, C., \& Sekuler, R. (2015). Obligatory and adaptive averaging in visual short-term memory. Journal of Vision, 15(4), 13. doi:10.1167/15.4.13

Dubé, C., Zhou, F., Kahana, M. J., \& Sekuler, R. (2014). Similarity-based distortion of visual short-term memory is due to perceptual averaging. Vision Research, 96, 8-16. doi:10.1016/j.visres.2013.12.016

Fouriezos, G., Rubenfeld, S., \& Capstick, G. (2008). Visual statistical decisions. Perception \& Psychophysics, 70(3), 456-464. doi:10.3758/PP.70.3.456

Gil-Gómez de Liaño, B., \& Botella, J. (2011). Concurrent memory load can help make RSVP search more efficient. Psicologica, 32, 13-30.

Gobell, J., \& Carrasco, M. (2005). Attention alters the appearance of spatial frequency and gap size. Psychological Science, 16(8), 644651. doi:10.1111/j.1467-9280.2005.01588.x

Haberman, J., \& Whitney, D. (2012). Ensemble perception: Summarizing the scene and broadening the limits of visual processing. In Wolfe, J., \& Robertson, L. (Eds.), From perception to consciousness: Searching with Anne Treisman: Oxford University Press.

Helson, H. (1947). Adaptation level as a frame of reference for prediction of psychological data. American Journal of Psychology, 60, 1-29. doi:10.2307/1417326

Hollingworth, H. L. (1910). The central tendency of judgment. Journal of Philosophy, Psychology, and Scientific Methods, 7, 461-469. http://www.jstor.org/stable/10.2307/2012819

Holt, L. L. (2006). The mean matters: Effects of statistically defined nonspeech spectral distributions on speech categorization. Journal of the Acoustical Society of America, 120(5), 2801-2817. doi:10.1121/1.2354071

Huang, J., \& Sekuler, R. (2010). Distortions in recall from visual memory: Two classes of attractors at work. Journal of Vision, 10(2), 24. doi:10.1167/10.2.24

Laing, E. J. C., Liu, R., Lotto, A. J., \& Holt, L. L. (2012). Tuned with a tune: Talker normalization via general auditory processes. Frontiers in Psychology, 3, 203. doi:10.3389/fpsyg.2012.00203

Loftus, G. F., \& Masson, M. E. J. (1994). Using confidence intervals in within-subject designs. Psychonomic Bulletin \& Review, 1, 476490. doi:10.3758/BF03210951

McElree, B. M., \& Dosher, B. A. (1989). Serial position and set size in short term memory: Time course of recognition. Journal of Experimental Psychology: General, 118, 346-373. doi:10.1037/0096-3445.118.4.346

Morey, C. C., \& Cowan, N. (2005). When do visual and verbal memories conflict? The importance of working-memory load and retrieval. Journal of Experimental Psychology: Learning, Memory, and Cognition, 31(4), 705-713. doi:10.1037/0278-7393.31.4.703

Onyper, S. V., Zhang, Y. X., \& Howard, M. W. (2010). Someor-none recollection: Evidence from item and source memory. Journal of Experimental Psychology: General, 139(2), 341-64. doi:10.1037/a0018926

Oriet, C., \& Brand, J. (2013). Size averaging of irrelevant stimuli cannot be prevented. Vision Research, 79, 8-16. doi:10.1016/j.visres.2012.12.004

Pestilli, F., \& Carrasco, M. (2005). Attention enhances contrast sensitivity at cued and impairs it at uncued locations. Vision Research, 45(14), 1867-1875. doi:10.1016/j.visres.2005.01.019

Piazza, E., Sweeny, T. D., Wessel, D., Silver, M., \& Whitney, D. (2013). Humans use summary statistics to perceive auditory sequences. Psychological Science, 24(8), 1389-1397. doi:10.1177/0956797612473759

Poulton, E. C. (1979). Models for biases in judging sensory magnitude. Psychological Bulletin, 86(4), 777-803. doi:10.1037/0033-2909.86.4.777 
Rissman, J., Gazzaley, A., \& D’Esposito, M. (2009). The effect of non-visual working memory load on top-down modulation of visual processing. Neuropsychologia, 47(7), 1637-1646. doi:10.1016/j.neuropsychologia.2009.01.036

Robitaille, N., \& Harris, I. M. (2011). When more is less: Extraction of summary statistics benefits from larger sets. Journal of Vision, 11(12), 18. doi:10.1167/11.12.18

Ross, J., \& Di Lollo, V. (1971). Judgment and response in magnitude estimation. Psychological Review, 78(6), 515-527. doi: $10.1037 / \mathrm{h} 0031784$

Simons, D. J., \& Myczek, K. (2008). Average size perception and the allure of a new mechanism. Perception \& Psychophysics, 70(7), 1335-1336. doi:10.3758/PP.70.7.1335

Smilek, D., Dixon, M. J., \& Merikle, P. M. (2006). Revisiting the category effect: The influence of meaning and search strategy on the efficiency of visual search. Brain Research, 1080(1), 73-90. doi:10.1016/j.brainres.2005.07.079

Smilek, D., Enns, J. T., Eastwood, J. D., \& Merikle, P. M. (2006). Relax cognitive strategy influences visual search. Visual Cognition, 14(4/5/6/7/8), 543-564. doi:10.1080/13506280500193487
Solomon, J. A., Morgan, M., \& Chubb, C. (2011). Efficiencies for the statistics of size discrimination. Journal of Vision, 11(12), 13. doi: $10.1167 / 11.12 .13$

Spencer, J. (1961). Estimating averages. Ergonomics, 4(4), 317-328. doi: $10.1080 / 00140136108930533$

Tuerk, A. S. (2014). Continuities and discontinuities in working memory representations of collections over ontogeny. Doctoral dissertation, Harvard University. http://nrs.harvard.edu/urn-3:HUL. InstRepos: 13129561

Van Selst, M., \& Jolicoeur, P. (1994). A solution to the effect of sample size on outlier elimination. Quarterly Journal of Experimental Psychology, 47A(3), 631-650. doi:10.1080/14640749408401131

Ward, L. (1987). Remembrance of sounds past: Memory and psychophysical scaling. Journal of Experimental Psychology: Human Perception and Performance, 13(2), 216-227. doi:10.3758/BF03210837

Watson, M. R., Brennan, A. A., Kingstone, A., \& Enns, J. T. (2010). Looking versus seeing: Strategies alter eye movements during visual search. Psychonomic Bulletin \& Review, 17(4), 543-549. doi:10.3758/PBR.17.4.543 